\title{
Temperature Dependence of Magnetization Process in Bistable Amorphous and Nanocrystalline FeCoMoB Microwires
}

\author{
P. Klein ${ }^{a}$, R. VARGA ${ }^{a}$ And M. VAZQUeZ ${ }^{b}$ \\ ${ }^{a}$ Institute of Physics, Faculty of Sciences, P.J. Safarik University, Park Angelinum 9, 04154 Kosice, Slovakia \\ ${ }^{b}$ Instituto de Ciencia de Materiales de Madrid, CSIC, 28049 Madrid, Spain
}

\begin{abstract}
We have studied the effect of thermal treatment on the magnetic properties of amorphous and nanocrystalline $\mathrm{Fe}_{40} \mathrm{Co}_{38} \mathrm{Mo}_{4} \mathrm{~B}_{18}$ microwires. The magnetization process was measured within the temperature interval from 80 to $425 \mathrm{~K}$. Microwires shows complex temperature dependence of the switching field in amorphous state due to the presence of complex stress distribution induced during production. After nanocrystallization, the switching field depends linearly on the measuring temperature that makes such microwires ideal for sensing applications.
\end{abstract}

PACS numbers: 75.50.Kj, 75.60.Ej, 75.60.Jk

\section{Introduction}

Amorphous glass coated microwires are ideal materials for new, modern microsensors [1]. Having positive magnetostriction, the microwires are characterized by a magnetic bistability due to their magnetization process that runs through the single Barkhausen jump when the external field exceeds the so-called switching field, $H_{\mathrm{sw}}$ [2]. Such bistability can be used in many applications like magnetic coding, sensors of magnetic field, mechanical stress, etc. However, the crucial parameter for their application is the time and temperature stability. Therefore, new classes of nanocrystalline microwires have been developed recently, having bistable behaviour, high Curie temperature and being structurally very stable $[3,4]$.

The temperature dependence of the magnetization process in glass-coated microwires has been studied by different authors $[5,6]$. The aim of this paper is to present a study of temperature dependence of magnetization process on different thermally treated amorphous and nanocrystalline $\mathrm{FeCoMoB}$ microwires.

\section{Experimental}

Amorphous glass-coated $\mathrm{Fe}_{40} \mathrm{Co}_{38} \mathrm{Mo}_{4} \mathrm{~B}_{18}$ microwires were produced by the Taylor-Ulitovski method. The diameter of metal core is $16 \mu \mathrm{m}$ and thickness of glass coating is $9 \mu \mathrm{m}$. The length of all samples used in the magnetization measurements was $1.5 \mathrm{~cm}$ and in the switching field measurement it was $10 \mathrm{~cm}$.

The microwires were annealed for $1 \mathrm{~h}$ at different temperatures in the range $300-825 \mathrm{~K}$ in order to obtain various stage of crystallization. The saturation magnetization was measured by the SQUID Magnetometer (Quantum Design) within the temperature range of $10-380 \mathrm{~K}$ in the magnetic field $1 \mathrm{~T}$. Temperature dependences of the switching field in the temperature range $80-425 \mathrm{~K}$ were measured by an induction method at a frequency of $50 \mathrm{~Hz}$.

\section{Results and discussions}

Due to the production process (rapid quenching, drawing), there is a complex stress distribution of the stresses induced in as-cast microwires. Therefore, the temperature dependence of switching field is also complex (Fig. 1). Firstly, it decreases at low temperature, then it rises, close to the $300 \mathrm{~K}$, showing discontinuos behaviour at high temperatures. Such temperature dependence can be understood taking into account the complex stress distribution and the additional stresses applied on microwire due to a different thermal expansion coefficient of metallic nucleus, $\alpha_{\mathrm{m}}$, and glass-coating, $\alpha_{\mathrm{g}}$. Varying the temperature of microwires, the complex stress distribution in as-cast microwires varies, too.

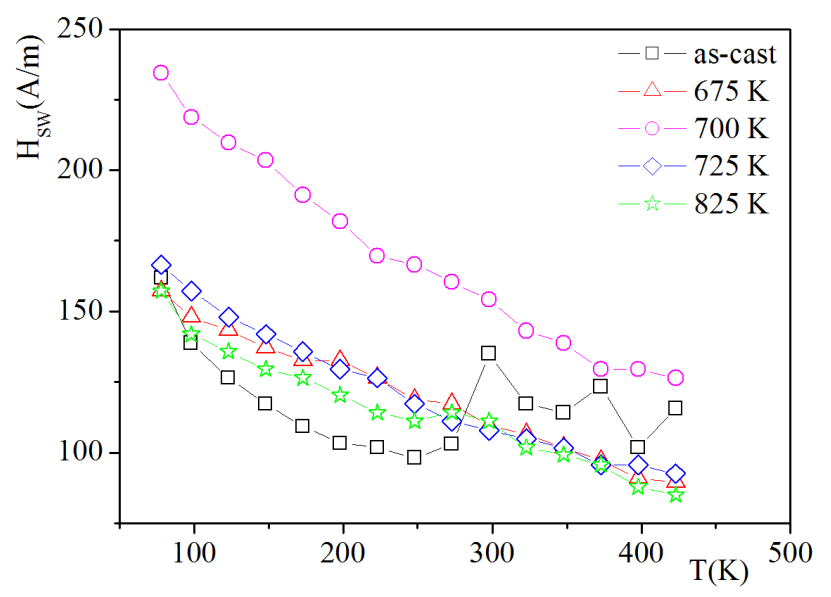

Fig. 1. Temperature dependence of switching field for heat-treated FeCoMoB microwire. Annealing temperature as a parameter.

Similar behaviour can be found from the measurement of saturation magnetization $M_{\mathrm{s}}$ (Fig. 2). Small disconti- 


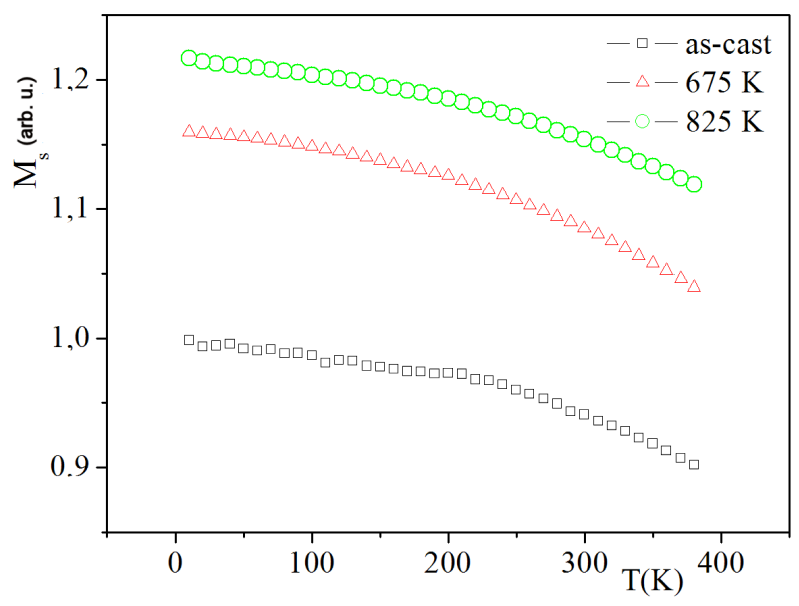

Fig. 2. Temperature dependence of saturation magnetization for heat-treated FeCoMoB microwire. Annealing temperature as a parameter.

nuities in the saturation magnetization are visible at low temperatures. The temperature dependence of magnetization is not smooth reflecting the changes in the complex stress distribution due to stress coming from glass coating. However, the applied field $1 \mathrm{~T}$ is strong enough to saturate the sample and such laborious behaviour is observed only below $200 \mathrm{~K}$. At higher temperature, the temperature dependence of $M_{\mathrm{S}}$ is smooth.

Annealing of microwires at $675 \mathrm{~K}$ leads to the stress relaxation and homogenization of the stress distribution in microwires. However, this temperature is still low enough for microwires to be amorphous [4]. The main anisotropy that determines magnetic properties of amorphous microwires is magnetoelastic one: $K_{\sigma} \sim \lambda_{\mathrm{s}} \sigma$ ( $K_{\sigma}$ is the anisotropy constant, $\lambda_{\mathrm{s}}$ being the magnetostriction, $\sigma$ being the applied stress). Hence the homogenization of the stress distribution leads to the smooth temperature dependence of the switching field. It decreases with a temperature following the decrease of the stress $\sigma$ applied on microwire by glass-coating due to the change of temperature $\Delta T: \sigma=E\left(\alpha_{\mathrm{g}}-\alpha_{\mathrm{m}}\right) \Delta T$ ( $E$ is the Young modulus). Moreover, the above-mentioned annealing (together with homogenization of the stress distribution) leads to the increase of $M_{\mathrm{s}}$, which has also very smooth temperature dependence.

Annealing at $700 \mathrm{~K}$ leads to an increase of the switching field. It was found that crystallization process starts close to this temperature [4]. However, crystalline precipitates are small, having a long distance between them. Hence, they act the role of the pinning centers for the domain wall and it results in the increase of $H_{\mathrm{sw}}$ (Fig. 1).

Annealing at the temperatures higher than $700 \mathrm{~K}$ leads to the appearance of nanocrystalline structure [4] that is characterized by the small crystalline grains (smaller than exchange length) embedded in amorphous matrix.
Exchange interaction of crystalline grains leads to the averaging out of magnetocrystalline anisotropy that results in a magnetic softness (switching field decreases). FeCoMoB composition was selected due to its positive magnetostriction in order to obtain bistable hysteresis loop. Such bistability was confirmed by measuring of hysteresis loops in [4]. Therefore, the temperature dependence of $H_{\mathrm{sw}}$ is driven mainly by magnetoelastic interaction of magnetic moments with the stress applied due to the glass-coating. The nanocrystalline FeCoMoB microwire is as soft as the amorphous one after the stress relaxation (by annealing at $675 \mathrm{~K}$ ) [4]. The advantage of FeCoMoB composition is that magnetic softness obtained by annealing is almost independent of the temperature of annealing within wide range of temperatures $725-875 \mathrm{~K}$. The temperature dependence of $H_{\mathrm{sw}}$ is also similar for different temperature of annealing within this range. However, its structural stability of nanocrystalline microwires is much higher even at high temperatures [4]. Moreover, the saturation magnetization of nanocrystalline microwires is higher than that of amorphous one (Fig. 2).

\section{Conclusions}

The temperature dependence of magnetization process in $\mathrm{FeCoMoB}$ amorphous and nanocrystalline glass-coated microwires has been studied. It was shown that nanocrystallization leads to the magnetic softness of such microwires. The temperature dependence of the switching field is smooth and almost linear and saturation magnetization increases by almost $25 \%$. This shows that the nanocrystalline $\mathrm{FeCoMoB}$ microwires are very promising materials for sensor applications.

\section{Acknowledgments}

This work was supported by the project NanoCEXmat No. ITMS 26220120019, Slovak VEGA grant No. 1/0076/09, MVTS 6RP/Manunet/UPJS/08 and project VVGS UPJS No. 30/09-10.

\section{References}

[1] M. Vázquez, Handbook of Magnetism and Advanced Magnetic Materials: Advanced Magnetic Microwires, Eds. H. Kronmüller, S. Parkin, Wiley, Chichester, UK 2007, p. 2193.

[2] H. Chiriac, T.A. Ovari, Prog. Mater. Sci. 40, 333 (1996).

[3] E. Komova, M. Varga, R. Varga, P. Vojtanik, J. Bednarcik, J. Kovac, M. Provencio, M. Vazquez, Appl. Phys. Lett. 93, 062502 (2008).

[4] P. Klein, R. Varga, P. Vojtanik, J. Kovac, J. Ziman, G.A. Badini-Confalonieri, M. Vazquez, J. Phys. D, Appl. Phys. 43, 045002 (2009).

[5] M. Vázquez, A.P. Zhukov, K.L. Garcia, K.R. Pirota, A. Ruiz, J.L. Martinez, M. Knobel, Mater. Sci. Eng., 375-377, 1145 (2004).

[6] A. Zhukov, Appl. Phys. Lett. 78, 3106 (2001). 